\title{
On the selection of excitation signals for the fast spectroscopy of electrical bioimpedance
}

\author{
Jaan Ojarand ${ }^{1,2}$ and Mart Min ${ }^{1}$ \\ 1. Thomas Johann Seebeck Department of Electronics, Tallinn University of Technology, Tallinn, Estonia \\ 2. E-mail any correspondence to: jaan.ojarand@ttu.ee
}

\begin{abstract}
Different excitation signals are applicable in the wideband impedance spectroscopy in general. However, in electrical bioimpedance (EBI) measurements, there are limitations that set specific demands on the properties of the excitation signals. This paper compares the efficiency of different excitation signals in a graspable presentation and gives recommendations for their use. More exactly, the paper deals with finding the efficient excitation waveforms for the fast spectroscopy of electrical bioimpedance. Nevertheless, the described solutions could be useful also in other implementations of impedance spectroscopy intended for frequency domain characterization of different objects.
\end{abstract}

Keywords: Impedance measurement; bioimpedance; signal design; signal to noise ratio; optimization

\section{Introduction}

Electrical impedance spectroscopy is a widely used tool for characterization the structure of tissues and cell cultures [1]. In the cases where the properties of objects are changing in time (e.g., heart muscle) or the objects are moving as cells in a microfluidic channel, the coverage of the frequency range of interest within a short timeframe demands to satisfy the criteria of the linear time-invariant (LTI) system. If the properties of a sample under test (SUT) are changing significantly during a measurement timeframe, the corresponding spectra will be distorted. Recently, a novel method was proposed for the measurement of periodically time-varying impedances using multisine excitation [2]. However, the most of impedances do not change periodically. For example, the impedance of cells moving fast between the electrodes of high throughput microfluidic system or the impedance of bacteria attaching to the electrodes of a biosensor, also the impedance of food products changing during their processing. That is, the short timeframe of every single nonrepeatable measurement becomes valuable.

As a result, the limited amount of energy of excitation signal spreads between multiple signal components with different frequencies during a short timeframe. Therefore, the power of corresponding individual components, equal to the square of their root-mean-square (RMS) values, decreases. The task to use the limited energy resources of signals most effectively and flexibly becomes necessary.

Concurrently, such an important criterion of the efficiency of impedance measurements - the signal-tonoise ratio (SNR) of measured signal - is proportional to the power of every frequency components in it, decreases as well. In electrical bioimpedance (EBI) measurements, unfortunately, the SNR cannot be improved by increasing the overall amplitude of the summary excitation signal, since it is limited to low values because of both two issues: a) satisfying the criteria of linearity of the LTI systems and

b) fulfilling the security needs for living tissues [3], [4].

Even for non-biologic measurements, the allowable input signal range and power supply voltage of electronic components, both limit the allowed amplitude of signals. Moreover, due to the high crest-factor (CF) of noisy signals, the probability of saturation of measurement channels increases. That leads to nonlinearities, which involve corresponding uncertainties into measurement results.

As discussed below, there are still several possibilities to improve the SNR in EBI measurements by optimizing the 
properties of excitation signals and specifying some parameters of the measurement system.

\section{Properties of the spectroscopy system}

The structure of the EBI spectroscopy system

The typical structure of EBI spectroscopy system is given in Fig 1. An excitation signal source (generator of the excitation waveform) has inputs for setting the required timing, frequency range, and amplitude. The signal from this generator provides an input excitation for the complex impedance to be measured $\dot{Z}$.

Next block, performs both, signal conditioning (amplification, filtering, normalizing) and analog-to-digital $(A / D)$ conversion. A part of this block (front end) is often located near the SUT for obtaining better performance.

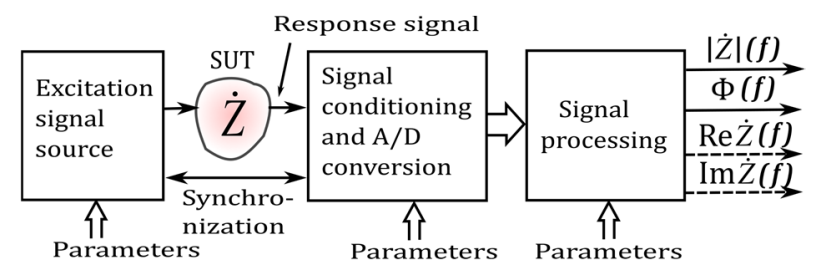

Fig.1: Simplified structure of the EBI spectroscopy system.

Discrete or fast Fourier transform (DFT or FFT) of the response signal from the SUT provides the amplitude $|\dot{Z}|(f)$ and phase $\Phi(f)$ spectra. Also the spectra of real $\operatorname{Re} \dot{Z}(f)$ and imaginary $\operatorname{Im} \dot{Z}(f)$ parts of the impedance $\dot{Z}$ can be calculated. Multichannel synchronous detection is an alternative to the Fourier transform if the number $k$ of frequency components is low. Later, here we use also notation $X[k]$ for the discrete spectrum and Mag $X[k]$ for the magnitude spectrum of spectral components $k$.

More exactly, to measure an unknown impedance, we can choose one of the two approaches. Either we apply a known voltage excitation across the SUT and measure the current response flowing through, or we inject a known current excitation into that object and measure the voltage response across it. We can also measure both, current and voltage, simultaneously. The last method is preferable if the excitation source is not stable enough in a required frequency range or the parasitic impedances cause too large changes on the known parameters of the excitation signal [5].

\section{Criterions of the efficiency of EBI spectroscopy system} Typically, the signal-to-noise ratio (SNR) of the measured signal is considered as the main efficiency criterion of the measurement system. The SNR of the EBI spectroscopy system depends on several factors, including the frequency bandwidth and measurement time. These parameters may be as well considered as efficiency criterions.

In several cases, e.g., for implantable and wearable measurement devices, the energy consumption, and physical dimensions are important. Also the reduced complexity of the measurement system is required frequently.

In turn, the parameters as frequency bandwidth, measurement time, and SNR of measurements are mutually related.

\section{Frequency range of EBI spectroscopy system}

To characterize the properties of biological objects, the $\alpha$ and $\beta$-dispersion frequency ranges are to be covered, usually. In the single-cell cytometry, capacitance and conductivity of the cell membrane and conductivity of the cytoplasm influence mainly the $\beta$-dispersion area that exhibits a characteristic frequency between several $\mathrm{kHz}$ up to tens of $\mathrm{MHz}$ [5]. In the case of living tissues, the spectrum of bio-impedance shifts toward lower frequencies and the spectra of the $\beta$-dispersion range cover typically 3-4 decades within the $\mathrm{kHz}$ to $\mathrm{MHz}$ band [1], [6]. However, even if only the $\beta$ dispersion area is of interest, some part of lower frequency area must be included since the $\alpha$ dispersion changes the plateau, from which the $\beta$ dispersion begins.

Frequency and time domain properties of signals Periodicity of signals and synchronous measurement Presentation of signals can be converted mutually between time and frequency domains by the aid of mathematical operators known as transforms. The use of Discrete Fourier Transform (DFT) or more efficient Fast Fourier Transform (FFT) for the conversion from time to frequency domain is essential in fast impedance spectroscopy. The inverse transforms (DFT ${ }^{-1}$ and $\mathrm{FFT}^{-1}$ ) apply to opposite conversions. Though the signals are classified as periodic and aperiodic ones, however, the aperiodic signals can be addressed also as periodic having infinitely long period in the time domain. In the frequency domain, such approach produces infinitely small frequency steps leading to a continuous spectrum. In signal processing practice, a signal component with the longest but finite period $T_{m}$ produces a discrete spectrum with frequency steps $f_{1}=1 / T_{m}$, where $f_{1}$ is the lowest frequency presented as a fundamental frequency component. A single one-shot event in one domain reflects as the periodic component in the opposite domain [7]. Even if the real signals are not periodic, DFT interprets them as periodic, which leads to the distortions of spectra (spectral leakage error). A signal $x(t)$ is periodic if:

$$
x(t)=x\left(t+T_{p}\right)
$$

The smallest positive value of $T_{p}$, which satisfies the condition (1) is a period. To be sure in signal periodicity, it must be observed from infinite past to the infinite future. Fortunately, this is not necessary, if the periodicity of signals is ensured by the design of the impedance spectroscopy system. 
Due to the discrete nature of signal processing, one more condition must be fulfilled for the synchronous DFT: the measurement interval $T_{m}=N \times t_{\mathrm{s}}$ must be an integer multiple of the period $T_{p}$ of the signal, where $N$ is the length of DFT. That is, the total number of samples $N$ used for calculation of Fourier transform, where $t_{\mathrm{s}}$ is a used sampling period. If the conditions of the synchronous measurement are not met, then a certain spectral leakage errors occur.

The spectral leakage errors can be reduced by employing special window functions in the time domain. However, this increases the complexity of signal processing and produces additional error components. Hence, if feasible, the synchronous measurement is always preferred.

\section{Minimal measurement interval and observation time} The measurement interval, $T_{m}$, which corresponds to the one period of the signal, determines the lowest frequency $f_{1}$ of the spectra obtained by DFT, $f_{1}=1 / T_{m}$.

Although only one period of a periodical signal fulfills the requirement of synchronous DFT $\left(T_{m}=T_{p}\right)$, more periods allow noise reduction through averaging. When the noise is not correlated neither with the signal nor with itself across trials, averaging yields an improvement of the SNR. If $\sigma$ is the amount of RMS noise in a set of $p$ samples, the averaged RMS noise is

$$
\sigma_{a v g}=\sigma / \sqrt{p}
$$

However, this improvement comes at the cost of increased total measurement time (observation time) $T_{o}$, $T_{o}=T_{m} \times p$. If the noise is correlated, the improvement is less effective.

The minimal measurement interval $T_{m}$ depends on the lowest frequency in the frequency range of interest. The frequency range, in turn, depends on the properties of SUT as will be explained in the next section. If the properties of SUT are varying in time, the total measurement interval should be decreased for the better conformity with the criteria of the time-invariant system.

One possibility is to decrease the observation time $T_{o}$ by reducing the number of measurement samples $p$. However, this also decreases the SNR. Another, better solution is to minimize time delays required for the formation of the response signal. Each time, when the excitation signal is applied, some settlement time of the response signal, depending mostly on the time-constants of the SUT, is required. Thus using the excitation signal that contains all the required frequencies (a multifrequency signal) provides minimal total observation time.

However, multifrequency signals have a drawback: their crest factor (CF) - the ratio of signal's peak value in respect to its RMS level - is often higher in comparison with a single frequency signal (sine wave). Moreover, even if the
CF of the multifrequency signal is similar or even below the CF of a single sine wave $(\sqrt{2})$, the RMS values of single multifrequency components are always less. RMS values of multifrequency components are decaying proportionally to the number of components $k$, as will be discussed in next sections of the paper.

The highest frequency in spectra and sample rate The highest frequency $f_{k}$ of the spectra is determined by the length $N$ of DFT, $f_{k}=f_{1} \times N / 2$. If the frequency bandwidth of measured signals is wider than $f_{k}$, then aliasing errors occur, because the signal power at higher frequencies mirrors at the lower frequencies. To minimize this distortion of spectra in the frequency range of interest, the sampling frequency $f_{s}=1 / t_{s}$ should be set high enough - typically 4 to 10 times above the highest frequency (though theoretically only 2 times). Unfortunately, this also requires more high-speed DSP components and increases the number of DFT samples.

\section{Normalization of signals and their energy content} To improve the SNR, higher RMS magnitudes of the frequency components of interest $(\mathrm{FCl})$ are desirable. For a graspable comparison of different waveforms, some variables must be normalized as constants. It is reasonable to normalize the amplitude to the unit value \pm 1 and the duration to 1 second. Normalizing the duration of the signal against 1 second also provides a normalized presentation of the spectra since the fundamental frequency is reciprocal to the duration $T_{p}$.

In general, RMS magnitudes of the frequency components (bins) of excitation signals are not equal. The shape of a magnitude spectrum may also be decaying, rising or oscillating around some mean value. The mean value of the RMS magnitudes of a number of frequency bins that fall in the half-power limit range is used here as a figure of merit for this general case.

According to Parseval theorem, the total energy in the frequency domain must be the same as calculated in the time domain. In the case of using DFT, this relation is following:

$$
\sum_{i=0}^{N-1}(x[i])^{2}=\frac{2}{N} \sum_{i=0}^{N / 2}(\operatorname{Mag} X[k])^{2},
$$

where $x[i]$ is a time domain signal with $i$ running from 0 to $N-1$, and $X[k]$ is its modified spectrum with $k$ running from 0 to $N / 2$. The modified spectrum is found by taking the DFT of the signal and dividing the first and last samples (samples 0 and $N / 2$ ) by the square root of two [7].

Single frequency excitation and spectrally dense wideband excitation signals

Single frequency signal and a short pulse

The only signal with a single line spectrum is the sine wave. Sinusoidal signal $x(t)$ can be described mathematically as: 


$$
x(t)=A \sin (2 \pi f t+\Phi)
$$

where $A$ is the amplitude and $\Phi$ is the initial phase, which specifies the waveform at time $t=0$. (A cosine wave is also "sinusoidal" but with a phase-shift of $\pi / 2$ ). If the SUT and measurement channel are linear, the output signal will also be a sinusoidal signal with the same frequency as the input. The drawback of using the single sine wave excitation is that several measurement steps at a number of frequencies are necessary and some settlement time is required in each sequential frequency step.

The infinitely short $\delta$-pulse can be considered as an ideal excitation signal for the fast impedance spectroscopy since it covers all the frequencies in the shortest time frame. However, in practice, there is a crucial drawback because the excitation pulses are very short ones with limited amplitudes, the energy of pulse spread over a number of frequency components, will be very low. The reason is that according to the left side of (3), the energy content of the signal is proportional to the surface area of the waveform, and in the frequency domain, this energy is divided among spectral components. In the case of very short pulses, the distribution of frequencies is dense, almost all the higher harmonics of the first frequency $f_{1}$ are present. If the amplitudes of the waveform are fixed (normalized), then the energy content of quadrangle pulses is directly proportional to their duration. If the duration of the pulses is increased, the magnitudes of frequency components are almost equal only in a shorter frequency range as illustrated in Fig. 2, and the mean value of their RMS magnitudes is nearly twenty times less compared to the RMS magnitude of a single sine wave.

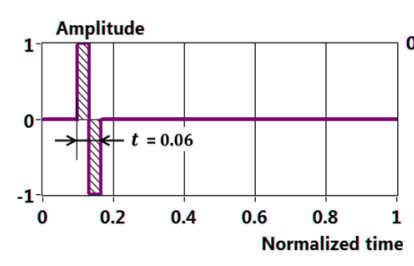

(a)

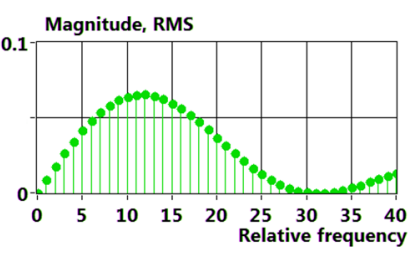

(b)
Fig.2: Waveform (a) and magnitude spectrum (b) of the pulse with relative duration $t=(64 / 1000)$.

Other types of short pulses, e.g., the chirps as shorttime frequency sweeps, could also be used for EBI measurements [8]. Chirps with a single cycle or even less can be generated, too. The RMS values of the first six components of the exponentially modulated short chirp [9] (Fig. 3) are higher in comparison with the pulses shown in Fig.2, but still low since the surface area of the waveform (hatched), being proportional to the energy content of the signal, is small.

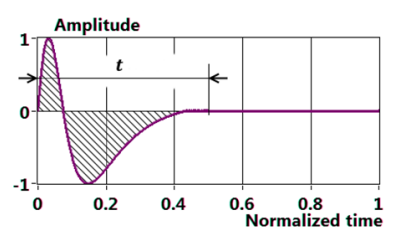

(a)

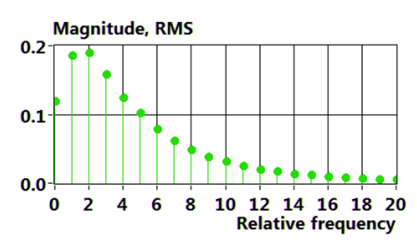

(b)
Fig.3: Waveform (a) and magnitude spectrum (b) of the exponentially modulated short chirp with relative duration $t=0.5$.

\section{Pulse waveforms with a longer duration}

The further symmetrical extension of the excitation signal shown in Fig. 2, runs out into a rectangular waveform in Fig. 4. In this case, the energy content of the signal is maximal. However, the spectrum contains only odd harmonics $\left(1^{\text {st }}\right.$, $\left.3^{\text {rd }}, 5^{\text {th }}, 7^{\text {th }}\right)$, which are declining fast with frequency by $1 / f$ rule. Such a simple waveform can be used, therefore, only in the cases where this type of decaying shape of the spectrum is useful. For example, a decaying part of the response current spectrum may be equalized with a similar decaying spectrum of the excitation voltage.

Another type of signal that covers a large number of harmonic frequencies with almost equal amplitudes is a step wave (Fig. 5). This type of excitation is often used in a time domain based impedance measurement [5], [10]. The total energy content of the signal starting from zero is near maximal, but there are also serious drawbacks. At first, most of the energy of the signal is concentrated in a DC component that is mostly undesired in EBI measurements. If the rise time of the step waveform tends to zero, the number of harmonics tends to infinity, but their magnitudes reach to zero. In practice, the rise time of the step waveform is larger than a zero moment, and the amplitudes of higher harmonics are decaying.

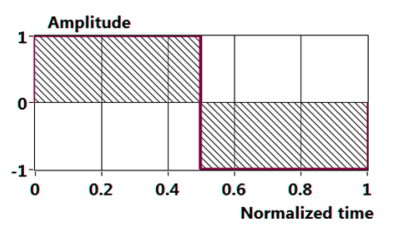

(a)

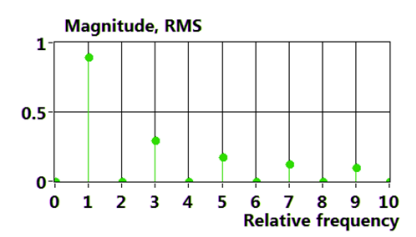

(b)
Fig.4: Rectangular waveform (a) and its magnitude spectrum (b).

A step waveform with the rise time of $1 / 500$ of the period $T_{p}$ is shown in Fig. $5 a$, where the RMS magnitudes of spectral components are almost constant up to $100^{\text {th }}$ higher harmonic but very low. DC component equals 0.9985. DC component of the signal can be canceled by shifting the starting point of the waveform near to -1 (dashed line in Fig. 5a), but the RMS values of other frequency components remain the same (very small). 


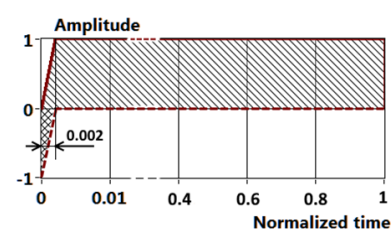

(a)

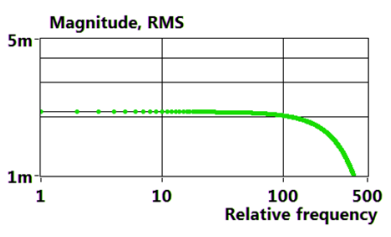

(b)
Fig.5: Waveforms (a) and magnitude spectrum (b) of the step waveform with a rise time of $1 / 500$ of the signal period. Note that the first part of the time scale is magnified by 20 . Diagonal hatch illustrates the energy content of the signal starting from zero and cross-hatches the energy content of the signal starting from -1 .

The energy content of the shifted waveform is proportional to a cross-hatched area, and its spectral content is similar to very short pulses.

The DC component can be canceled also by adding a second part of opposite polarity. However, the waveform resumes in near rectangular signal with a well known decaying shape of the magnitude spectrum (Fig. 4). It must be emphasized here, that DFT interprets the signal as periodic, and according to (1) all the changes of the waveform must be included in a period.

The situation described here also illustrates the drawback of time-domain impedance measurement method with a short pulse or step waveforms. In the case of unbalanced step waveforms, most of the excitation signal energy is useless for spectroscopy (concentrated in a DC component). Faster measurement cycle comes at the cost of lower SNR since the energy of harmonic spectral components is created only during the front part of the step or duration of the balanced pulses. Another reason for the low level of spectral components is that the energy spreads over many harmonic components. Averaging improves the SNR, however, this contradicts with the idea of fast measurements. Moreover, the use of longer excitation with a higher level of desired spectral components is more efficient under similar conditions since only uncorrelated noises can be effectively suppressed by averaging.

\section{Chirps}

Chirps are considered here as signals of many cycles of sinusoidal oscillation with a changing "instantaneous" frequency. A sine-wave chirp signal with an instantaneous phase $\theta(t)$ can be described mathematically as

$$
S_{c h}(t)=\sin (\theta(t))=\sin \left(2 \pi \int f(t) d t\right)
$$

with the „instantaneous“ frequency

$$
f(t)=(\theta(t) / d t) / 2 \pi
$$

In practice, however, generating of discrete signals is more convenient. The waveforms of the sinusoidal and discrete, so-called signum-chirp $S_{s g n}(t)=\operatorname{sign}\left(S_{c h}(t)\right)$ are shown in Fig. $6 \mathrm{~b}$.

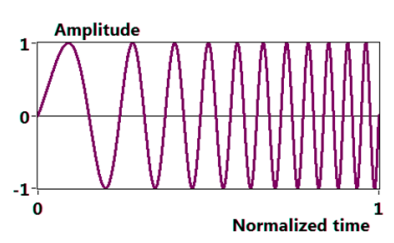

(a)

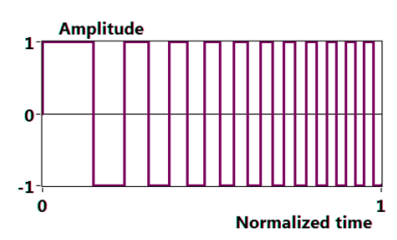

(b)
Fig.6: Waveforms of the sinusoidal (a) and signum-chirp (b).

The dependency of the mean RMS magnitudes on a number of frequency bins $k$ of chirp signals is described in [11]. RMS magnitudes of spectral components of normalized linear sinusoidal chirp tend to the value of $1 / \sqrt{k}$ and are higher by a factor of $4 / \pi$ in the case of signum chirp.

Further analysis shows some deviation (ripple) of the RMS magnitudes $x$ of frequency components from a mean value of all used components $k$. The ripple in per cents is calculated as

$$
D=\left(\left(\left(x_{\max }-x_{\min }\right) / 2\right) / x_{\text {mean }}\right) \times 100
$$

and is presented in Fig. 7 and Fig. 8.

The advantage of signum chirp (in comparison with a sinusoidal one) is higher RMS value of its spectral components, but the ripple is also considerably higher in the cases, when the number of desired harmonic components, $k>10$.

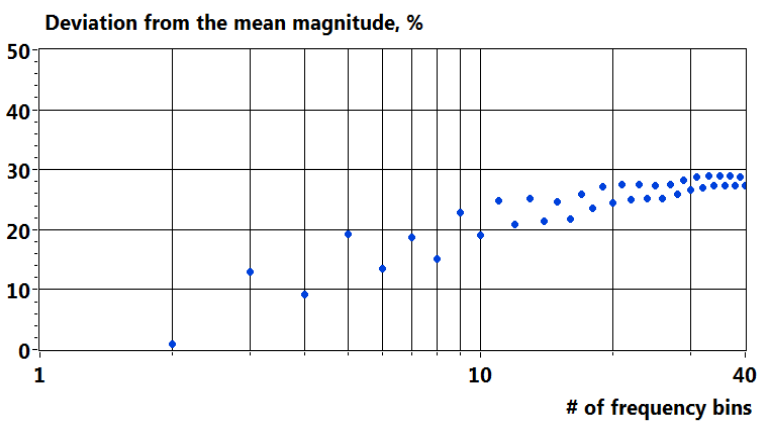

Fig.7: Relative deviation of RMS magnitudes of the frequency components from their mean values of the linear sinusoidal chirp with normalized frequencies in the range from 10 to 50 .

Another disadvantage of signum chirp is that some part of the energy, typically $15-20 \%$ of total energy, always spreads into higher harmonics above $k$. 


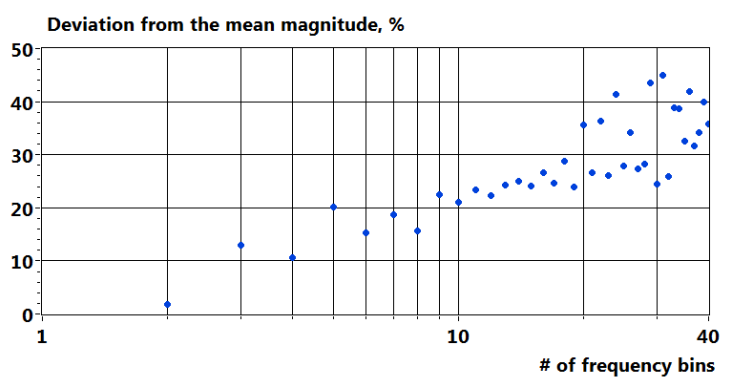

Fig.8: Relative deviation of RMS magnitudes of frequency components from their mean values of the signum chirp with normalized frequencies in the range from 10 to 50 .

\section{Maximum length binary sequence, MLBS}

Another type of wideband excitation waveform with a dense distribution of frequencies is a maximum length sequence (MLBS). MLBS is a type of pseudorandom binary sequence, which can be easily generated by an $n$-stage linear feedback shift register (LFSR). A maximum-length LFSR produces a maximum length sequence ( $m$ sequence). It cycles through all possible $N=2^{n}-1$ states within the $n$ stage shift register (except the state where all bits are zero). The MLBS period $T_{p}=\left(2^{n}-1\right) \times t_{c}$, where $t_{c}$ is the period of the register clock. The RMS magnitude spectrum follows the envelope of $\sin (f) / f$ function as illustrated in Fig. 9. The half-power bandwidth occurs at a frequency of approximately $0.443 / t_{c}[12]$.

The shape of the magnitude spectrum becomes flattered as $N$ increases, and more frequency bins fall in the half-power limit range. However, the power of each frequency component decreases. We showed in [11] that the mean RMS magnitudes of the frequency components of the MLBS signal tend to the value

$$
\operatorname{Mag} X_{0.5}=B \frac{4}{\pi} \frac{A}{\sqrt{2}} \frac{1}{\sqrt{k_{h}}},
$$

where $\mathrm{B} \approx 0.93$ and $k_{h}$ is a number of useful frequency bins that fall in the half-power limit range.

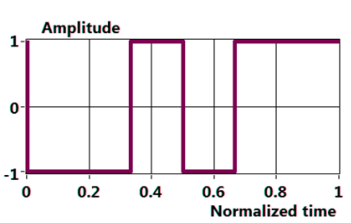

(a)

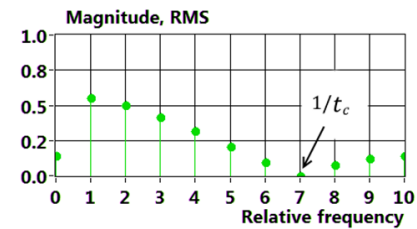

(b)
Fig.9: Waveform of the 3-rd order MLBS (a) and its magnitude spectrum (b).

It may be concluded that the magnitudes of the spectral components of MLBS and signum chirp signals depend almost similarly on a number of frequency components $k$. Even so, there are also important differences. At first, the number of spectral components of the MLBS signal is fixed by its order $n$, and secondly, a shape of the spectrum is smoother with a fixed relative deviation near $\pm 15 \%$ from its mean value (later defined by the half-power limit).

\section{Spectrally sparse wideband excitation signals \\ Distribution of frequencies and the power of spectral components}

The excitation signals described above (except sinewave) have a dense distribution of frequencies. Spreading of the signal energy in such a way reflects in a lower power of separate frequency components. If the frequency response of SUT is smooth, as in the case of EBI measurements, the concentration of the signal energy into a limited number of components is more adequate than the dense distribution. A more detailed illustration will be given in next sections.

The accuracy of fitting the equivalent circuit model with the impedance measurement results depends on the number of frequency components. In general, the use of more frequencies improves the fitting accuracy [13]. However, the fitting accuracy also depends on the signal to noise ratio (SNR) of measurements. As already discussed in previous sections, the magnitudes of spectral components of the excitation signal are decreasing, when their number increases. It follows that the number of used spectral components has two contradictory effects on the accuracy of estimation of parameters of the equivalent circuit model. Usually, less than ten components are required in EBI spectroscopy.

\section{SNR and shape of the magnitude spectrum}

The SNR is expressed through the ratio of signal and noise power $P_{\text {signal }} / P_{\text {noise }}$, while the power is equal to the square of RMS value. Assuming that the power density of noise is uniform in the frequency range of interest, the SNR of spectral components is proportional to the square value of their RMS levels. Since the magnitude of the impedance spectrum of biological objects decays with frequency, the spectrum of the excitation signal should have such a shape that elevates the response signal at higher frequencies. In the case, when polarizable electrodes are used, the shape of the excitation signal should provide a lower level response at lower frequencies, too.

If the noise power density is not uniform in the frequency range of interest, then the better overall SNR is obtained when the shape of the excitation spectrum follows the power density spectrum of noise. Moreover, in-vivo electrical bioimpedance (EBI) measurements must comply with the safety regulations. The safe applied current for the BF and CF type devices is limited according to the IEC60601 standard.

In EBI measurements, the required dynamic range of the impedance magnitude may reach three decades and more [1], [5]. Therefore adaption of the magnitudes of spectral components of the excitation provides better SNR. From the excitation signals described above, only the sine 
wave and sinusoidal multicycle chirp allow free adjustment of the magnitudes of separate frequency components.

\section{Binary multifrequency signals (BMS)}

Binary multifrequency waveforms in Fig. 10a and Fig. 11a with two discrete values ( \pm 1 , if normalized) look similar to the maximum length sequence (MLBS) shown in Fig. 9a. However, a sequence of pulses in the period $T_{p}$ of binary multifrequency waveforms is not distributed by the rule used for generating the MLBS waveforms.

According to the Fourier principle, periodic signals can be represented by a set of discrete frequencies. A spectrum of the periodic BMS waveform (a pattern of pulse sequence) may be composed as the sum of spectra of its $L$ components with different durations:

$$
X_{B M S}=\sum_{i=1}^{L} X(i) .
$$

Magnitude spectrum $X(i)$ of individual waveform components $i$ depends only on their duration (amplitudes are normalized), but their contribution into the overall magnitude spectrum of the BMS waveform also depends on their phase spectra. Phase spectra of the waveform components, in turn, depend on their polarity and position in a signal period. Choosing appropriate lengths and positions of the waveform components allow designing the BMS with different magnitudes of its spectral components, including the zero and near-to-zero values.

As a result, the shapes of the BMS spectra differ principally from the envelope of sinc-function being characteristic to MLBS. The envelope of the spectrum lines of synthesized BMS may have as decaying, as well as flat (Fig. 10b) or even rising shape (Fig.11b).

The CF of the BMS waveforms is the smallest of possible $(C F=1)$, and the total energy of BMS excitation is two times higher compared to the sine or multi sine wave with the same amplitude. Considering the simplicity in generating binary signals, BMS also meets the criteria of reduced energy consumption and lower complexity. The disadvantage of the BMS is that some part of the energy always spreads into higher harmonics outside the desired spectral range. Therefore, characterization of the BMS only by the smallest CF = 1 is an overestimation of its merits.

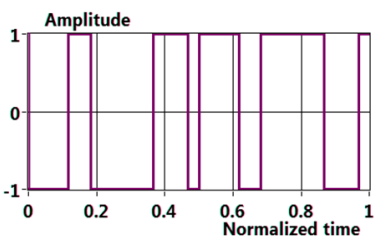

(a)

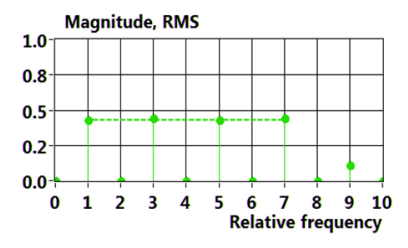

(b)
Fig.10: BMS waveform (a) and its magnitude spectrum (b) with four equally emphasized components (frequency bins 1, 3, 5, 7).

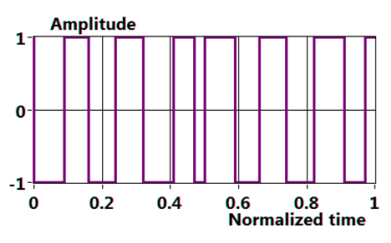

(a)

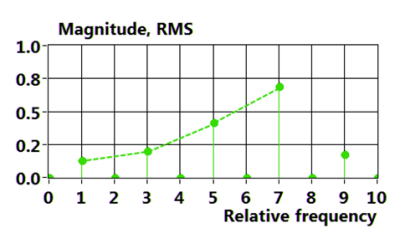

(b)
Fig.11: BMS (a) and its magnitude spectrum (b) with rising levels of components (frequency bins 1, 3, 5, 7).

\section{Multisine excitation}

Multisine excitation retains the advantages of the sine wave while reducing the measurement time. The specific advantage of the multisine signal is that only the desired components appear in the spectra. Adding up several ( $k$ ) sine waves leads to decreasing the individual spectral components in the excitation signal because its overall amplitude is limited, moreover, their RMS values decrease proportionally to CF, as we showed in [11]:

$$
S_{R M S}(i)_{M a x|s(t)|=1}=\frac{1}{\sqrt{k} \times C F}
$$

Let us indicate here that the CF of the sum of same level consequent frequency components with optimal phases can be below the corresponding value of a single sine wave ( $C F=\sqrt{2}$ ) [4]. Our optimization algorithms provide CF below this value for a consequent frequency distribution $i=1,2,3,4 \ldots k$ and $k>6$, Fig. 12 . The enhanced algorithm described in [14] gives CF below 1.37 at large $k$.

Let us take an example. In the case of 36 added components $\mathrm{CF}=1.358$. If the total amplitude of the sum of signals with equal amplitudes is set to $1 \mathrm{~V}$, then according to (10), RMS values of all components are $122.8 \mathrm{mV}$ that is 5.76 times less than RMS value of the single sine wave with the amplitude of $1 \mathrm{~V}$.

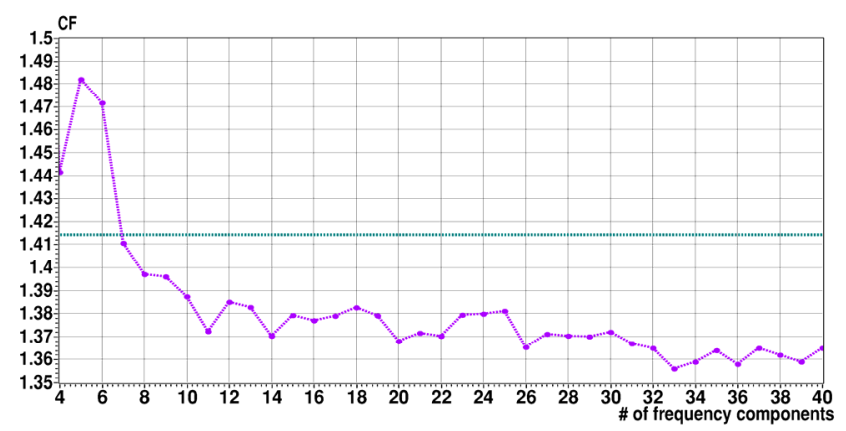

Fig.12: CF of the optimized multisine signal with a consequent frequency distribution $(i=1,2,3,4 \ldots . k)$, for a $k$ in the range from 4 to 40 . A green line level corresponds to the CF of a single sine wave.

In the case of very sparse distribution of frequencies, the optimization of initial phases of multisine components is ineffective, and the CF tends to its worst possible case 
$(\sqrt{2 k})$. However, in the case of less sparse frequency distributions as a binary logarithmic one, the results prove to be acceptable. For example, the CF of the multisine signal with binary logarithmic distribution at the number of components $k=16(i=1,2,4,8,16,32, \ldots, 32768)$ tends to the value $\pi \sqrt[3]{k} / 2 \sqrt{2}$. Moreover, a multisine with the binary distribution of spectral components does not require optimization of initial phases since their zero value provides near optimal results [15].

Remark: even though some part of the BMS energy transfers to useless higher harmonics, the RMS levels of the components of well optimized BMS are higher than of the optimized multisine. A benefit of using BMS increases with the sparsity of frequency distributions. Fig. 13 illustrates RMS magnitudes of both excitation waveforms for the different frequency distributions.

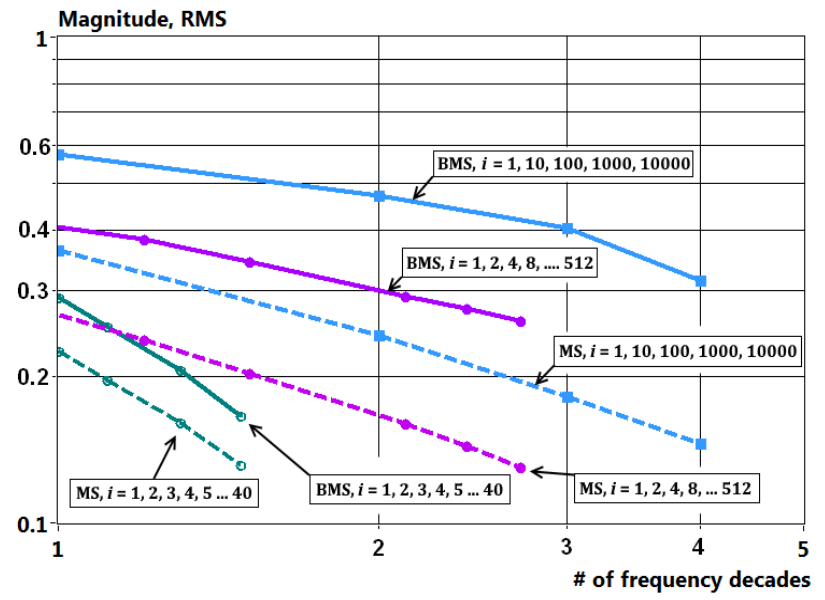

Fig.13: Normalized RMS magnitudes of consecutively and logarithmically distributed frequency components of optimized multisines (MS, dashed lines) and BMS (solid lines) vs. a number of frequency decades a signal covers.

\section{Multilevel binary signals}

The stair-step multifrequency waveforms (MFS) [16] and tri-level (ternary) waveforms (MFM) [17] are recently proposed for the broadband bioimpedance spectroscopy. Although these waveforms can be used as multifrequency excitation, there are also some limitations and disadvantages.

Calculations with (3) show that the total energy content of described 6-th order MFS and MFM waveforms are 61\% and $83 \%$, respectively, in comparison with two level BMS. Taking into account that $83.6 \%$ of the energy of the MFM signal falls into the desired frequency components, the overall energetic efficiency is $69.3 \%$. The typical energetic efficiency of the BMS signal depends on the distribution of frequencies and is in the range from 65 to $85 \%$.

Limitations of the described MFM waveforms are that only binary distribution of frequencies and decaying shapes of magnitude spectra are produced. MFS waveform provides a flat magnitude spectrum, but its total energy content is significantly lower in comparison with MFM and BMS. Furthermore, generation of MFM and MFS waveforms requires more resources in comparison with BMS.

\section{Ethical approval}

The conducted research is not related to either human or animal use.

\section{Discussion}

There are several criteria for rating the efficiency of EBI spectroscopy system as described in the second section. The criteria are related and are often contradictory, e. g., as the SNR and speed of measurements. A choice of proper excitation signal has an important role in acceptable satisfying of different requirements.

Even though the short signals cover wide bandwidth, they do not suit well for EBI spectroscopy. Since the amplitude of signals is strictly limited, the energy content of short pulses (proportional to the waveform area) remains low. In other terms - the crest factor CF of this type of signals is high (too high). The second disadvantage is that the energy of short pulses is spread widely over too many frequency components. The third drawback is that the amplitudes of individual spectral components are not freely adjustable.

Disadvantages of the step waveform have common roots with short pulses. If the step starts from zero, most of the energy is concentrated into a DC component, and the magnitudes of other spectral components are low. Though the shifted initial level cancels the DC component, the remaining waveform becomes similar to a short pulse.

Binary waveforms (MLS, signum chirp, and BMS) have the lowest crest factor ( $C F=1$ ) that guarantees highest energy content of the excitation signal in a measurement interval in comparison with other waveforms. The simplicity in generating of binary signals also meets the criteria of reduced energy consumption and lower complexity.

A common disadvantage of all the binary excitations is that some part of the energy always spreads onto higher harmonics of the desired frequency components. This part is mirrored at lower frequencies and distorts the spectra.

Another important aspect is that the overall SNR of impedance measurements also depends on the CF of the response signal. The use of binary waveforms may significantly increase it in comparison with the sinusoidal waveforms [18]. Limiting the bandwidth of binary excitation improves the situation. However, simple filtering also produces distortions and increases the CF of the excitation signal. Another solution is increasing the sampling rate, but this raises both the complexity and energy consumption.

Additional disadvantages of the MLS and signum chirp excitations are that the energy spreads onto too many frequency components, and the amplitudes of spectral components are not freely adjustable. BMS does not have 
these drawbacks. However, the adjustment range of magnitudes is limited, especially if the rising shape of the spectrum is desired.

Tri-level and stair-step multifrequency waveforms do not have clear advantages - their CF is significantly higher in comparison with BMS, but generation requires more resources.

The advantage of the multisine waveform is that only the desired components appear in the spectra, and the amplitudes of spectral components are freely adjustable. The disadvantages of multisine (in comparison with the $\mathrm{BMS}$ ) are higher CF, especially in the case of sparse frequency distributions, and increased complexity of generation. However, in the cases where the higher accuracy of measurements is required, it is a good choice. Besides, the multisine waveforms can be used also for the composition of near-binary waveforms [18]. The CF of such signals is near 1.2 even in case of sparse frequency distributions and the distortions caused by unwanted higher frequency components of binary waveforms are eliminated.

To decrease the dynamic range of amplitudes, the use of several time-separated excitation signal sections with different frequency subranges may be an optimal solution.

\section{Conclusion}

In electrical bioimpedance (EBI) measurements, several limitations set specific demands on the properties of the excitation signals. Signal-to-noise ratio (SNR), measurement time, and the complexity of realization were considered as the main criteria of efficiency.

Multicomponent signals, both multisine and binary (BMS), have a clear advantage over the spread spectrum ones (short pulses, step waveform, binary chirps, pseudorandom sequences). The final choice is as follows the multisine wave excitation gives the highest accuracy, but the multi-frequency binary excitation enables the lowest complexity.

The accuracy of fitting of the equivalent circuit impedance model with the impedance measurement results depends on the number of used frequency components. An optimal number of frequencies of the multifrequency excitation is a topic for further research.

\section{Conflict of interest}

Authors state no conflict of interest.

\section{References}

1. Grimnes, S. and Martinsen, Ø. G. 2015. Bioimpedance and bio-electricity basics. 3rd edition. Elsevier-Academic Press.

2. Sanchez, B., Louarroudi, E., and Pintelon, R. 2015. Timeinvariant measurement of time-varying bioimpedance using vector impedance analysis. Physiol. Meas. 36, pp. 595-620. https://doi.org/10.1088/0967-3334/36/3/595
3. Pliquett, U. 2010. Bioimpedance: A review for food processing. Food. Eng. Rev. 2, 2010, pp. 74-94. https://doi.org/10.1007/s12393-010-9019-z

4. Ojarand, J. and Min, M. 2014. Crest factor optimization of the multisine waveform for impedance spectroscopy bioimpedance spectroscopy. Physiol. Meas. 35, pp. 10191033. https://doi.org/10.1088/0967-3334/35/6/1019

5. Min, M., Parve, T. and Pliquett, U. 2015. Impedance detection. In: Prof. Dongqing Li ed. Encyclopedia of microfluidics and nanofluidics. $2^{\text {nd }}$ edition. New York: Springer, pp. 1338-1361. https://doi.org/10.1007/978-14614-5491-5_1783

6. Schwan, H. P. 1963. Determination of biological impedances. In: Nastuk W.L. ed. Physical techniques in biological research. New York: Academic Press. 6, pp. 323-406. https://doi.org/10.1016/B978-1-4831-6743-5.50013-7

7. Smith, S. 1999. The Scientist and engineer's guide to digital signal processing. San Diego: California Technical Publishing.

8. Nahvi, M. and Hoyle, B. 2009. Electrical impedance spectroscopy sensing for industrial processes. IEEE Sensors Journal. 9, pp. 1808-1816. https://doi.org/10.1109/JSEN.2009.2030979

9. Ojarand, J. et al. 2010. Nonlinear chirp pulse excitation for the fast impedance spectroscopy. Electronics and Electrical Engineering. 100(4), pp. 73-76.

10. Pliquett, U. 2013. Time-domain based impedance measurement: strengths and drawbacks. Journal of Physics: Conference Series 434, pp. 1-4. https://doi.org/10.1088/1742-6596/434/1/012092

11. Ojarand, J., Rist, M., Min, M. 2016. Comparison of excitation signals and methods for a wideband bioimpedance measurement. In: Proc. of IEEE International Instrumentation and Measurement Technology Conference (I2MTC 2016), Taipei, Taiwan, May 23-26. Hoboken, NJ, USA: IEEE Conference Publications, pp. 1291-1296. https://doi.org/10.1109/I2MTC.2016.7520555

12. Godfrey, K. R. 1991. Introduction to binary signals used in system identification. Proc. Int. Conf. Control. 1, pp. 161-166.

13. Ward, L. and Cornish, B. 2004. Multiple frequency bioelectrical impedance analysis: how many frequencies to use? In: Proc. ICEBI XII and EIT V. 61, pp. 321-324.

14. Ojarand, J. and Min, M. 2017. Recent advances in crest factor minimization of multisine. Electronics and Electrical Engineering. 23(2), pp. 59-62. https://doi.org/10.5755/j01.eie.23.2.18001

15. Ojarand, J. et al. 2014. Optimization of multisine excitation for a bioimpedance measurement device. In: IEEE International Instrumentation and Measurement Technology Conference (I2MTC 2014), Montevideo, Uruguay, May 12-15. Hoboken, NJ, USA: IEEE Conference Publications, pp. 829832. https://doi.org/10.1109/I2MTC.2014.6860859

16. Yang, Y. et al. H. 2014. Development of a stair-step multifrequency synchronized excitation signal for fast bioimpedance spectroscopy. BioMed Research International. 2014, pp. 1-8.

17. Yang, Y. et al. 2015. Design of tri-level excitation signals for broadband bioimpedance spectroscopy. Physiol. Meas. 36, pp. 1995-2007. https://doi.org/10.1088/0967-3334/36/9/1995

18. Ojarand, J. and Min, M. 2014. Efficient excitation signals for the fast impedance spectroscopy. Electronics and Electrical Engineering. 20(5), pp. 144-149. https://doi.org/10.5755/j01.eee.20.5.7115 